\title{
Impact of Iron Fortification on Anaemia and Iron Deficiency among Pre-school Children Living in Rural Ghana
}

Samuel Kofi Tchum ( $\nabla$ kofi.tchum@kintampo-hrc.org )

Kintampo Health Research Centre and Kwame Nkrumah University of Science and Technology, College of Science, Department of Biochemistry and Biotechnology https://orcid.org/0000-0003-4800-1769

\section{Fareed Kow Arthur}

Kwame Nkrumah University of Science and Technology, College of Sciences, Department of Biochemistry and Biotechnology, Kumasi

\section{Bright Adu}

University of Ghana, Noguchi Memorial Institute for Medical Research, College of Health Sciences, Department of Immunology, Legon, Accra

\section{Samuel Asamoah Sakyi}

Kwame Nkrumah University of Science and Technology, School of Medical Sciences, Department of Molecular Medicine, Kumasi

\section{Latifatu Alhassan Abubakar}

Kintampo Health Research Centre, Kintampo-North

\section{Dorcas Atibilla}

Kintampo Health Research Centre, Kintampo-North

\section{Seeba Ibn Amenga-Etego}

Kintampo Health Research Centre, Kintampo-North

\section{Felix Boakye Oppong}

Kintampo Health Research Centre, Kintampo-North

\section{Francis Dzabeng}

Kintampo Health Research Centre, Kintampo-North

\section{Benjamin Amoani}

University of Cape Coast, School of Allied Health Sciences, Department of Biomedical Sciences, Cape Coast

\section{Thomas Gyan}

Kintampo Health Research Centre, Kintampo-North

\section{Kwaku Poku-Asante}

Kintampo Health Research Centre, Kintampo-North

\section{Research}


Keywords: Anaemia, Iron deficiency, Micronutrient powder, Iron deficiency anaemia

Posted Date: May 27th, 2020

DOI: https://doi.org/10.21203/rs.3.rs-30758/v1

License: (c) (1) This work is licensed under a Creative Commons Attribution 4.0 International License. Read Full License 
1 Impact of Iron Fortification on Anaemia and Iron Deficiency among Pre-school Children Living in Rural Ghana

3

4

21 Email: kofi.tchum@kintampo-hrc.org. Phone: +233 244923774 Benjamin Amoani ${ }^{5}$, Thomas Gyan ${ }^{1}$ and Kwaku Poku-Asante ${ }^{1}$ Sciences, University of Cape Coast, Cape Coast, Ghana. North, Bono East, Ghana.
Samuel Kofi Tchum ${ }^{1,2^{*}}$, Fareed Arthur ${ }^{2}$, Bright $\mathrm{Adu}^{3}$, Samuel Asamoah Sakyi ${ }^{4}$, Latifatu Alhassan Abubakar ${ }^{1}$, Dorcas Atibilla ${ }^{1}$, Seeba Amenga-Etego ${ }^{1}$, Felix Boakye Oppong ${ }^{1}$, Francis Dzabeng ${ }^{1}$,

${ }^{1}$ Department of Biochemistry and Biotechnology, College of Sciences, Kwame Nkrumah University of Science and Technology, Kumasi, Ghana; ${ }^{2}$ Kintampo Health Research Centre, Kintampo-North, Ghana; ${ }^{3}$ Department of Immunology, College of Health Sciences, Noguchi Memorial Institute for Medical Research, University of Ghana, Legon, Accra, Ghana; ${ }^{4}$ Department of Molecular Medicine, School of Medical Sciences, Kwame Nkrumah University of Science and Technology, Kumasi, Ghana; ${ }^{5}$ Department of Biomedical Sciences, School of Allied Health

*Corresponding Author: Samuel Kofi Tchum, Kintampo Health Research Centre, Kintampo- 


\section{Abstract}

\section{Background}

29 Micronutrient interventions, principally vitamin A and zinc supplementation for children, and

30 fortification of foods with iron and iodine, are considered the most cost-effective global

31 development efforts. Multiple micronutrient powder is a mixture of at least iron, zinc and vitamin

32 A used to prevent malnutrition in children and during health emergencies. Micronutrient

33 deficiencies are a universal health burden among young children in developing countries.

34 However, the use of this low cost but sustainable micronutrient powder as an innovative home-

35 fortification approach to control a common nutritional disorder like iron deficiency anaemia

36 among pre-school children living in malaria endemic sub-Saharan Africa is unclear. The aim of

37 our study was to determine the effect of providing long-term continued prophylactic micronutrient

38 powder with iron on the risk of iron deficiency and anaemia among pre-school children living in

39 rural Ghana.

40 Methods

41 This population-based randomized-cluster trial was conducted in the Bono region of Ghana from

$423^{\text {rd }}$ April to $6^{\text {th }}$ July 2010. 1958 children were recruited, and 967 randomly assigned to receive

43 prophylactic micronutrient powder with iron and 991 assigned to receive placebo. The trial

44 participants were children aged between 6 to 35 months, identified at home and able to eat semi-

45 solid foods (with or without breast milk). Structured questionnaires were administered, their blood

46 samples were also taken for biochemical analysis. They were randomly assigned to receive daily

47 micronutrient powder without or with iron $(12.5 \mathrm{mg})$ added to complementary meals immediately

48 after enrollment for five months. Each participant also received anti-malaria treated bednet and

49 chemotherapy. Weekly follow up visits were conducted at home or health facility where data on 
50 malaria using rapid diagnostic test and hospital admissions were collected. The primary outcome

51 was post supplementation of prophylactic micronutrient powder with iron to mitigate the effects

52 of iron deficiency and anaemia.

\section{Results}

541958 children were recruited and 967 randomly assigned to receive prophylactic micronutrient 55 powder with iron and 991 assigned to receive placebo. Loss to follow up was $7 \%$ (143), with vital 56 status at 35 months of age reported for 1904 (97.2\%). Anthropometry, anaemia, iron status, 57 demographic characteristics and dietary intakes were similar between the groups at baseline.

58 Baseline haemoglobin level was significantly higher compared to haemoglobin level at endline ( $\mathrm{p}$ $59<0.0001)$. Though, we recorded an increase in haemoglobin $(\mathrm{p}=0.0001)$ and ferritin $(\mathrm{p}=0.0002)$

60 levels in the iron group than in the placebo group at the end of the intervention. Soluble transferrin

61 receptor levels were more saturated among children from the iron group compared to placebo 62 group $(\mathrm{p}=0.012)$. Anaemic status in the iron group improved compared to the non-iron group ( $\mathrm{p}$ $63=0.03)$.

\section{Conclusion}

65 The risk of childhood morbidity and mortality in rural Ghana is high, mainly due to iron deficiency

66 anaemia. National nutritional policy coupled with the current WHO recommendations are required

67 to support the provision of prophylactic micronutrient powder with iron in order to improve 68 anaemic and iron status among pre-school children in rural Ghana.

70 TRIAL REGISTRATION clinicaltrials.gov Identifier: NCT01001871. Registered $27^{\text {th }}$ October 71 2009, http://www. ClinicalTrials.gov/ NCT01001871

72

73 Keywords: Anaemia, Iron deficiency, Micronutrient powder, Iron deficiency anaemia. 


\section{Introduction}

75 The global occurrence of anaemia for pre-school children is $43 \%$ of which about $42 \%$ is

76 attributable to iron deficiency [1]. Early childhood anaemia diminishes cognitive ability and causes

77 developmental delays and disability [1,2]. Zinc deficiency is alleged to be as prevalent as iron

78 deficiency affecting about 293 million children below five and is accountable for $13 \%$ of lower

79 respiratory tract infections [3]. Multiple micronutrient powder (MNP) is a mixture of at least iron,

80 zinc, and vitamin A used to prevent malnutrition in children and during health emergencies [4].

81 Micronutrient deficiencies are a universal health burden, particularly for young children in

82 developing countries [5]. Micronutrient interventions, principally vitamin A and zinc

83 supplementation for children, and fortification of foods with iron and iodine, are considered the

84 most cost-effective global development efforts [6]. MNP with iron given to children improve

85 motor and cognitive performance and mitigate severe anaemia prevalence $[7,8]$ but has no effect

86 on malaria morbidity and mortality if anti-malarial interventions were available $[9,10]$.

88 Little data exist on whether given long-term continued prophylactic MNP with iron to children

89 aged 6 to 35 months living in malaria endemic sub-Saharan African countries will reduce iron

90 deficiency anaemia (IDA). Moreover, the risk of iron deficiency (ID) and anaemia among pre-

91 school children living in these countries remain a major public health threat [11]. Data obtained

92 from 1993 to 2005 indicated that iron deficiency was prevalence in more than $24 \%$ of the global

93 pre-school children population $[1,12]$. In sub-Saharan Africa, the problem was worsened by the

94 coexistence of preventable anaemia and malaria resulting in increased childhood morbidity and

95 mortality [13, 14]. Previous studies involving motor and mental (social and cognitive)

96 development among pre-school children have indicated the need for early prevention of childhood 
97 anaemia through innovative but sustainable iron intervention programmes because such poor

98 motor and cognitive skills have been associated with moderate anaemia (haemoglobin $<100 \mathrm{~g} /$

99 L) and might be irreversible [15-17].

100

101 In 2003, a randomized placebo-controlled trial conducted in Pemba, Zanzibar involving 32,000

102 pre-school Tanzanian children was stopped promptly on the advice from the trial's Data Safety

103 Monitoring Board (DSMB) due to higher hospitalizations or mortality rate in the iron groups [10].

104 However, a further secondary subgroup analysis involving a recruited iron-replete children at

105 baseline (BL) discovered a limitation on the risk of adverse events, which led to ethical difficulties

106 and complicated study designs in malaria endemic areas [10]. The UNICEF and WHO joint

107 statement was uncertain about MNP use, since the absorption characteristics differ considerably

108 from iron syrups or tablets if given to children aged between 6 and 36 months [18]. In 2006, the

109 joint statement was amended to specifically recommend home fortification of foods plus MNP

110 with iron to children at risk of iron deficiency and anaemia [19]. Finally, based on substantive

111 findings, the WHO in 2016 recommended that in heavily malaria transmission areas, pre-school

112 children at risk of iron deficiency and anaemia should be provided with oral iron intervention if

113 they have access to anti-malaria intervention strategies (insecticide-treated bednets, anti-malarial

114 drug therapy and vector-control programmes [3]. Further studies are therefore needed to answer

115 the question of whether continued long-term prophylactic iron fortification might improve intrinsic

116 iron and anaemic status among pre-school children living in malaria endemic regions. Thus, the

117 overall aim of this study was to determine the effect of providing long-term continued prophylactic

118 MNP with iron on the risk of iron deficiency and anaemia among pre-school children living in

119 rural Ghana. 


\section{Subjects and Methods}

\section{Study Area}

123 Our trial was conducted in Wenchi Municipality and Tain District in the Bono Region of Ghana.

124 In 2010, the combined population for the two contiguous areas was 198,125. A total of 11,215 pre-

125 school-aged children, representing nearly $0.3 \%$ of total children under five in Ghana were living

126 in the two districts [20]. A total of 8,548 compounds in 99 smaller communities existed in Wenchi

127 Municipality $(\mathrm{n}=89,739)$ and Tain District $(\mathrm{n}=108,386)$ [20]. Rains in the trial areas start from

128 April to November with a mean rainfall per annum of $1250 \mathrm{~mm}$ plus an average temperature range

129 from $18-38{ }^{\circ} \mathrm{C}$ [21]. This period is characterized by high malaria transmission. In Ghana, malaria

130 is mostly caused by $P$. falciparum with an estimated $2 \cdot 3$ million cases in 2017 , thus making it

131 holoendemic [22]. In 2003, anaemia prevalence among pre-school-aged children was 76.1\% (95

$132 \%$ C.I. $73 \cdot 9-78 \cdot 2 \%)[23,24]$.

\section{Study Design}

135 This study was a population-based randomized-cluster trial conducted in the Bono region of

136 Ghana. The trial participants were young children, identified at home and able to eat semi-solid

137 foods (with or without breast milk). For five months, all participants received daily micronutrient

138 powder without or with iron $(12.5 \mathrm{mg})$ added to complementary meals. However, children who

139 had severe anaemia (haemoglobin $<70 \cdot 0 \mathrm{~g} / \mathrm{L}$ ), severe malnutrition (weight-for-length z-score <

$140-3 \cdot 0)$, receipt of iron in supplements within the past 6 months or chronic disease were excluded.

141 In order to maximize the opportunity for optimum anaemia and iron status assessment, the study

142 occurred in the rainy season during high malaria transmission. 


\section{Ethical Issues and Trial Monitoring}

145 The ethics committees of the Ghana Health Service (GHS), Food and Drugs Authority (FDA) of

146 Ghana, Kintampo Health Research Centre (KHRC) and Hospital for Sick Children (SickKids)

147 Canada approved our trial. Registered ClinicalTrials.gov number was NCT01001871. The trial

148 was overseen by a Data and Safety Monitoring Board, which was constituted in October 2009 and

149 held three meetings during the course of the trial. Members of the board included international and 150 local health policy makers expertized in randomized controlled trials, nutrition, paediatrics,

151 statistics and social sciences. The board's statistician summarized the compiled outcome data at

152 the end of the recruitment phase and half-way via the intervention stage. The children's primary

153 caregivers consented to participate in the study. For the interim analysis, if there were any serious

154 adverse events (i.e. hospital admissions or deaths) in the iron group than the non-iron group, the 155 agreement a priori was that the study would be terminated.

\section{Recruitment of Subjects}

158 Participants aged between 6 to 35 months were enrolled from early April 2010 through to July 1592010 and randomly assigned (ratio 1:1) to receive either iron or no iron at the compound level with

160 the aid of computer-generated model. A cluster represents a compound which comprise of one or 161 more households living in the same residence with the resident families having at least one child 162 eligible for inclusion into the trial. In order to prevent cross contamination between the groups via 163 food sharing, a cluster randomization design was employed. Upon enrolment, each child was 164 provided with an insecticide treated net (ITN) and the caregiver was educated on its appropriate 165 usage. Sachets containing the powdered fortificant (MNP-Sprinkles ${ }^{\circledR}$ Mumbai, India) without or 
with iron were similar except a subtle 'A' or 'B' labelled markings and double-blinded to the

167 caregivers and study team.

169 The children from the iron group were provided a daily MNP dose containing elemental iron (12.5

$170 \mathrm{mg})$ in microencapsulated ferrous fumarate, vitamin A (400 $\mu \mathrm{g})$, ascorbic acid (30 mg) and zinc

171 (5 mg) [25, 26]. Similar fortificant without iron (Placebo) was provided to the children in the non-

172 iron group. Caregivers were all provided with MNP and instructed to mix the package's contents

173 with a small bit of semi-solid meals on daily basis. This dosing MNP regimen continued for 5

174 months and then the participants were further monitored an extra month without the powdered

175 fortificant. During the duration of the study, routine weekly household visits were conducted by

176 field researchers (FRS) to assess participants' health (including axillary temperature) and collected

177 data on MNP adherence, ITN use and morbidity. Caregivers were also advised to take their sick

178 or febrile children to the nearest health facilities for assessment and prompt treatment between the

179 routine visits.

\section{Specimen and Data Collection}

182 The participants' health was assessed at baseline (BL) and endline (EL) of MNP intervention

183 including body temperature. At BL and EL, $500 \mu \mathrm{L}$ blood sample was taken from the finger or heel

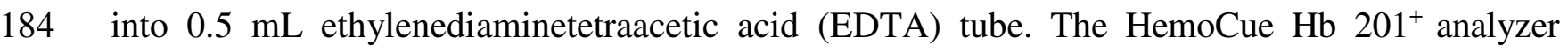

185 (HemoCue AB, Angelholm, Sweden) was used to measure the haemoglobin ( $\mathrm{Hb}$ ) levels and severely

186 anaemics were referred immediately. Preliminary rapid diagnostic test (RDT) (Paracheck Pf ®

187 Device, Orchid Biomedical Systems, Verna, Goa, India) for malaria was quickly done and those

188 confirmed positive for the test were treated for malaria. After recovery, participants were enrolled if 
189 all other inclusion criteria were met. At the laboratory, haematological, malaria microscopy, acute

190 protein phase and iron biomarkers were tested on the remaining blood. If a child is febrile (i.e.

191 axillary temperature $>37.5^{\circ} \mathrm{C}$ ) or febrile 48 hours ago, $100 \mu \mathrm{L}$ capillary blood sample was collected

192 into $0.5 \mathrm{~mL}$ EDTA tube for full blood count, malaria rapid and blood smear test (for parasitaemia 193 and speciation) during the study as described in the following procedures [26].

195 Specimen Processing and Analysis

196 Thick and thin blood films were prepared and the thin films fixed with methanol. Both smears

197 were then geisma-stained. Each sample slide was read by two independent microscopists and if 198 discrepancy between the two readers was over $50 \%$, a third microscopist was consulted [26]. The 199 confirmed malaria cases (RDT assay) were treated with artemisinin-based combination therapy 200 (ACT), a combination of Artesunate-Amodiaquine or Artemether-Lumefantrine administered as 201 the current first-line national antimalarial chemotherapy treatment [27]. To verify if treatment was 202 successful, the participants were monitored for 14 days after the first dose and on the $7^{\text {th }}$ and $14^{\text {th }}$ 203 day, malaria status was assessed using both RDT and microscopy. The haematology auto-analyzer 204 (Horiba ABX Micros 60-OT-CT-OS-CS, Montpellier, France) measured the full blood counts 205 (FBC). The QuikRead 101 analyzer (Orion Diagnostica, Espoo, Finland) immunoturbidimetrically 206 measured plasma C-reactive protein (CRP). Red blood cell zinc protoporphyrin (ZPP) was 207 measured using a haematofluormeter (Model 206D, Aviv Biomedical Inc., Lakewood, NJ, USA). 208 Indirect enzyme-linked immunosorbent assay (ELISA) measured plasma ferritin (Fn) (Spectro 209 Ferritin S-22, Ramco Laboratories Inc. USA) and transferrin receptor (TfR) (TFC-94, Ramco 210 Laboratories Inc. USA) levels as described in the following procedures [26]. 


\section{Outcomes}

213 Our primary outcomes were anaemia $(\mathrm{Hb}<100 \mathrm{~g} / \mathrm{L})$ and iron deficiency $(\mathrm{Fn}<30 \mu \mathrm{g} / \mathrm{L}, \mathrm{ZPP}>$

$21452 \mu \mathrm{mol} / \mathrm{mol}$ heme). Acknowledging that Fn and ZZP interpretation will be confounded by acute

215 phase response, we excluded these indicators for those children who had an elevated CRP (> $8 \mathrm{mg}$

216 / L) $[28,29]$. Secondary outcomes included clinical malaria, expressed as any parasitaemia level

217 including reported febrile or axillary temperature $>37 \cdot 5^{\circ} \mathrm{C}$ within 48 hours [30]. Certain medical

218 episodes such as malaria parasitaemia levels exceeding $5000 / \mu \mathrm{L}$, hospitalization as a result of

219 diarrhoea (three or more watery or loose stools within 24 hours), other pneumonia symptoms (such

220 as cough, tachypnea, lower chest wall indrawing and either pleural effusion or consolidation on a

221 chest X-ray) and finally, cerebral malaria or meningitis based on clinical judgement were

222 considered severe.

\section{Statistical Analysis}

225 Our hypothesis indicated that anaemia and iron deficiency prevalence rates would significantly 226 improve among the children from the Fe group than their non-Fe counterparts. Using a $67 \%$

227 reduction in anaemia prevalence as baseline rate [31], with power of $90 \%$ and $5 \%$ type I error, if

228 all exposed participants had the same level of risk at start of the trial, then we can assumed a $30 \%$

229 prevalence of anaemia among the placebo group at the end of MNP intervention. However, after

230 accounting for a loss of $15 \%$ to follow-up, a calculated sample size of 1940 participants (970 per

231 group) was used. Visual Fox Pro version 9.0 data management programme was used to double-

232 enter all clinical and epidemiological data for discrepancies, typographical errors and extreme

233 observations. Errors from the database were regularly verified with field staff and discrepancies

234 resolved prior to decoding the randomization and analyzing the data using STATA (Stata 
236 were used to summarize the study variables. The prevalence of anaemia, iron deficiency and iron

237 anaemia at the end of the study was reported with their 95\% confidence intervals. Using logistic

238 regression, the risk of anaemia, iron deficiency and iron deficient anaemia were compared between

239 the Fe and non-Fe group. Generalized estimating equation with robust standard errors was used to

240 obtain population-averaged estimates and to account for the household level clustering. Parameter

241 estimates were reported as odds ratio with their $95 \%$ confidence intervals Separate models were

242 considered for anaemia, iron deficiency and iron deficient anaemia. In all the models, we adjusted

243 for child's age ( $\leq 12$ months, $13-24$ months and $>24$ months) and sex. Also, we adjusted for

244 baseline anaemia, baseline iron deficiency and baseline iron deficient anaemia in the model for

245 anaemia, iron deficiency and iron deficient anaemia respectively. All analysis were carried out on

246 an intention-to-treat (ITT) basis.

\section{Results}

249 A total of 2220 children aged 6 - 35 months from 22 communities were screened for eligibility 250 from $3^{\text {rd }}$ April to $6^{\text {th }}$ July 2010 (Figure 1). Of these, 262 (11.8\%) were excluded according to pre251 specified criteria. A total of 1958 children were randomly assigned to receive either prophylactic 252 micronutrient powder $(n=967)$ or placebo $(n=991)$ (Figure 1$)$. Characteristics of the children 253 were similar between the groups (Table 1). By the end of the study ( 24 weeks), about $3.0 \%$ of the 254 participants were lost to follow-up $(\mathrm{Fe}=67$ versus non-Fe $=76)$ for $863 \cdot 8$ child years of total 255 observation time. The lost to follow-up in both groups was as a result of moved-outs from the trial 256 area. Adherence was similar between the groups (90.7\% for Fe versus $93.0 \%$ for non-Fe children) 257 and ITN use also did not differ (mean 91.9\%) (Table 1). 
At baseline, the mean $\mathrm{Hb}$ concentration was similar in both the Fe and non-Fe group (10.3 $\mathrm{g} / \mathrm{L}$ in

259 both groups, $p$-value $=0.69)($ Table 2$)$. Out of the 1958 children enrolled, $1806(92.2 \%)$ were

260 blood-sampled after the MNP intervention (Table 2). We also observed that, the mean Hb level in

261 both groups was significantly higher at baseline compared to endline $(\mathrm{p}<0.0001)$. However, the

262 mean $\mathrm{Hb}$ level after the intervention was significantly lower in the non-Fe group compared to the

263 Fe group $(9.3 \pm 1.5 \mathrm{~g} / \mathrm{L}$ versus $9.7 \pm 1 \cdot 7 \mathrm{~g} / \mathrm{L}$ respectively, $\mathrm{p}=0 \cdot 0001)$ (Table 2$)$.

265 Baseline prevalence of anaemia, iron deficiency and iron deficiency anaemia were similar in the

266 Fe and non-Fe group $(\mathrm{p}>0.05)$ but the endline prevalence of these iron indicators were

267 significantly improved in the Fe compared to the non-Fe children $(\mathrm{p}<0.05)$. Moreover, in Fe and

268 non-Fe groups, the endline prevalence rates of anaemia, iron deficiency and iron deficiency

269 anaemia were significantly improved compared to the baseline prevalence rates of these iron

270 indicators $(\mathrm{p}<0.05)($ Table 2$)$. Similarly, among children who were iron deficient at baseline $(\mathrm{n}=$

271 818), their mean ZPP concentration differences were greater in the non-Fe group (indicating

272 greater risk of iron deficiency) compared to the Fe group (11.9 $\pm 162 \cdot 0$ for Fe versus $9 \cdot 6 \pm 139 \cdot 3$

$273 \mu \mathrm{mol} / \mathrm{mol}$ of heme for non-Fe versus Fe children $)(\mathrm{p}<0 \cdot 0001)$. Paradoxically, almost all subjects

274 in both groups who provided blood samples at endline were iron deficient (mean 99.1\%), thereby,

275 rather grossly overestimating the prevalence of ID when compared with the conventional criteria

276 (> $52 \mu \mathrm{mol} / \mathrm{mol}$ haem) (Table 2). Of the children who had blood-sampled at endline, $52.7 \%$ were

277 moderately anaemic (47.8\% for Fe versus $52.2 \%$ for non-Fe children) and overall prevalence of

278 severe anaemia $(\mathrm{Hb}<70 \mathrm{~g} / \mathrm{L})$ was $6.0 \%$ (4.2\% for Fe versus $7.7 \%$ for non-Fe children). At

279 endline, the prevalence of anaemia was $58.6 \%(\mathrm{~N}=1059,95 \%$ CI: $56.3 \%-60.9 \%)$. 
281 The prevalence of moderate and severe anaemia were $52.7 \%(\mathrm{~N}=951,95 \% \mathrm{CI}: 50.3 \%-55.0$

$282 \%)$ and $6.0 \%(\mathrm{~N}=108,95 \% \mathrm{CI}: 5.0 \%-7.2 \%)$ respectively (Table 2). Iron deficiency was

283 prevalent in $24.5 \%(\mathrm{~N}=443,95 \% \mathrm{CI}: 22.6 \%-26.6 \%)$, while the prevalence of iron deficiency

284 anaemia was $13.5 \%(\mathrm{~N}=243,95 \%: 12.0 \%-15.1 \%)$ (Table 2). The prevalence of anaemia, iron

285 deficiency and iron deficiency anaemia by Fe and non-Fe group was presented in Table 3. From

286 the results of the risk adjusted logistic regression analysis, the odds of anaemia, iron deficiency

287 and iron deficiency anaemia was significantly higher in the children from the non-Fe group

288 compared to those in the Fe group (Table 3).

289

290 Discussion

291 The results of our study indicated that daily prophylactic micronutrient powder plus iron mixed

292 with a small bit of semi-solid meals to children aged 6 - 35 month increased haemoglobin levels,

293 improved anaemic and iron status in rural Ghana. These findings were consistent with studies that

294 reported improvement in iron deficiency and anaemia after prophylactic micronutrient powder

295 supplementation [7, 32, 33]. Our results were also similar to other MNP trials in Ghana [7, 26],

296 Gambia [34], Turkey [35] and Kyrgyzstan [36] that also observed improved haemoglobin levels

297 and iron status among young children on MNP supplements. The improved Hb levels and iron

298 status may be due to iron response to the hormone erythropoietin, that accelerated the production

299 of new erythrocytes via erythropoietic processes in the bone marrow [36]. In our study, we also

300 observed that the transferrin receptor levels were more saturated after the intervention among the

301 Fe-containing fortificant children than the placebo and this finding was consistent with other Fe-

302 supplementation studies that evidenced the benefit of daily Fe fortificants among pre-school 303 children $[35,37]$. 
305 Iron deficiency and IDA prevalence rates improved at the end of the intervention among the

306 children in the Fe group than those from the non-Fe group (Table 2). Moreover, the children who

307 were iron-deficient $(\mathrm{AOR}=1.68)$ and iron-deficient anaemics $(\mathrm{AOR}=2.12)$ in the Fe group were

308 more likely to recover from ID and IDA respectively compared to their counterparts in the non-Fe

309 group after the intervention. These findings were consistent with other Fe-MNP trials [38, 39].

310 Even though, some other studies associated ID and IDA prevalence rates equivocally with the

311 effect of MNP intervention [37, 40, 41]. Reasons for our observation, though poorly understood,

312 maybe attributed to the fact that $\mathrm{Fe}$ is the only micronutrient, homeostatically regulated via

313 absorption and the mechanism behind the iron-regulatory hormone, hepcidin is currently

314 incomprehensible. However, some others investigators have suggested that in the presence of

315 malaria and Fe fortificant, hepcidin may be upregulated to prompt dyserythropoiesis [42]. This

316 then deprived the malaria pathogens of circulating Fe, a source of nutrient for their survival in the

317 host [42]. The resultant increased Fe stored within ferritin in the hepatocytes via the transferrin

318 receptors may lead to the replacement of $\mathrm{Fe}$, a substrate for the enzyme ferrochelatase with zinc

319 and may elevate ZPP levels [43]. The directionality of these findings suggested a risk-lowering

320 effect of MNP [i.e. since the confidence interval favoured a lower risk ratio (RR)]. However, an

321 exploratory sub-group analysis of baseline haemoglobin and ZPP concentrations indicated that

322 children from the Fe group who were iron replete (ZPP $\leq 52 \mu \mathrm{mol} / \mathrm{mol}$ of heme) with moderate

323 anaemia (Hb 70 - $100 \mathrm{~g} / \mathrm{L})$ had a $25 \%$ lower risk of symptomatic malaria (RR 0.85, $95 \%$ C.I.

$3240.53-1 \cdot 36)$ and a $28 \%$ lower risk of severe malaria (RR 0.82, $95 \%$ C.I. $0.48-1 \cdot 39$ ) compared

325 to non-Fe children who were iron replete and moderately anaemic at baseline (data not shown).

326 Moreover, baseline moderate anaemia and iron deficiency were strongly associated with a $36 \%$ 
327 reduced risk of clinical (RR 0.73, $95 \%$ C.I. 0.56 - 0.94) and a $39 \%$ for severe malaria (RR 0.70,

$32895 \%$ C.I $0.52-0.95)$ than being iron replete and anaemics at baseline. These findings were

329 consistent with systematic reviews of 39 studies among 32,759 children on daily Fe supplement

330 living in malaria hyper-endemic areas [3]. Paradoxically, whole blood ZPP levels were limited in

331 discriminating between the participants with and without iron deficiency (Table 2). Even when

332 combined with haemoglobin levels, no added diagnostic value was observed but rather grossly

333 overestimated ID prevalence rate when compared to the conventional cut off points ( $>52 \mu \mathrm{mol} /$

334 mol haem). These findings from our study were consistent with other MNP studies that used ZPP

335 as additional iron indicator [44-46], but contrary to other MNP intervention findings too [46, 47].

336 This may have been attributed to the differences in ZPP cut-offs used to define iron deficiency,

337 which was lower in our study ( $>52 \mu \mathrm{mol} / \mathrm{mol}$ of heme) compared to Zanzibar (> $80 \mu \mathrm{mol} / \mathrm{mol}$

338 of heme) [10]. Several ZPP cut-offs for defining iron deficiency have been proposed on the basis

339 of the population group being studied and the specimen processing method (e.g. washed versus

340 unwashed red blood cells). The blood samples in our study were washed before being analyzed.

341 To our knowledge, this was not done in the Zanzibar trial and so a higher ZPP cut-off was observed

342 [48]. Despite this adjustment, however, differences in iron status classification between trials may

343 have affected the comparability of statistical outcomes. One main common finding between our

344 study and that of Zanzibar was the significant protective effect of iron to mitigate the risk of iron

345 deficiency and anaemia among the young children. In contrast, however, we did not find a

346 significant risk lowering MNP effect associated with baseline iron deficiency alone without 347 concurrent anaemia. 
349 We did not increased the risk to hospital admission at 35 months between the intervention group

350 and control arm. This differs from a similar trial in Zanzibar which reported an increase risk to

351 malaria related admissions among study children who received iron [10]. Other suggestions may

352 be that the lower risk to hospitalization in our study was due to anti-malaria ITNs and drug

353 chemotherapy interventions that were provided to the study children. In our study, hospitalization

354 rates were the same between the groups during or after the intervention ( $\mathrm{OR}=1 \cdot 20,95 \%$ C.I.

$3550.91-1.58 ; \mathrm{p}>0.05$ ). Moreover, the incidence of other clinical diagnoses (pneumonia, diarrhoea

356 or meningitis) among the hospitalized children were also similar between groups with or without

357 a concurrent malaria diagnosis ( $p>0.05$ ). In 2009, a systematic review of 14 studies reported that

358 the provision of iron mitigated the risk of clinical malaria but the effect was reversed when routine

359 malaria management and surveillance were absent [49]. However, none of the studies included

360 iron fortification intervention trials. It was also unclear whether the data on malaria morbidities

361 were obtained by parental report or whether the children were examined by trained fieldworkers.

362 Our study used powdered iron fortificant (ferrous fumarate) with different absorption

363 characteristics from the iron supplements (provided in the form of iron and folic acid tablets) used

364 in the Zanzibar trial. Furthermore, the microencapsulation of the iron (ferrous fumarate) protected

365 the iron in the food matrix from oxidation, which likely reduced and delayed peak plasma iron

366 concentrations [50-52]. This may have reduced the level of freely accessible iron in circulation

367 and mitigated the risk of malaria.

369 Our study was conducted in the rainy season when malaria transmission was high, resulting in

370 very dense malaria parasitaemia in both groups (Table 1) though, the Fe intervention had no

371 influence on the level of malaria parasitaemia [26]. Per-protocol analysis, (49\% of the Fe group, 
$372 \mathrm{n}=1023$ ) similar findings to the intent to treat (ITT) analysis in terms of the overall incidence of

373 clinical and severe malaria was observed. Contrary to the ITT analysis, however, the risk of clinical

374 malaria on the impact of the fortificant powder with iron was not modified by baseline iron

375 deficiency or anaemia status. Normally, as maternal passively-acquired immunity wanes in infants

376 and young children, malaria becomes a major contributor to anaemia due to direct haemolysis of

377 both parasitized and uninfected erythrocytes by the body's immune system and also temporary

378 bone marrow malfunction $[3,53]$. Other contributors of anaemia are consumption of semi-solid

379 weaning diet low in Fe that mostly contains non-bioavailable iron [54]. The high demand for more

380 dietary Fe during childhood development may be a limiting factor among infants and young

381 children from low income or poor homes [54]. After the study, a total of five deaths were recorded,

382 malaria and septicaemia accounted for three deaths (1 for Fe versus 2 for non-Fe children) and

383 both malnutrition with severe dehydration and road traffic accident (both Fe children) claimed one

384 life each according to reports from conducted medical and verbal autopsy. But these child 385 mortalities were not related to MNP intervention in our study. Yet these findings were not 386 influenced by the MNP intervention.

\section{Conclusion}

389 Our findings did not only addressed a research gap in knowledge but advocated an important 390 nutritional policy that will mitigate childhood iron deficiency and anaemia if implemented in iron 391 supplementation programme as a preventive strategy based on recommendations from WHO and 392 UNICEF [3, 18]. However, for ethical reasons, the provision of ITNs and prompt / appropriate 393 malaria treatment (whenever indicated) ensured that all the children benefited from the prevailing 394 malaria control activities. Therefore, we are confident that our results and the current WHO 
395 guidelines [3] recommending the use of MNP to treat and prevent iron deficiency and anaemia

396 among preschool children should rekindled the interest and advocacy to implement MNP use as a

397 national nutrition policy in malaria endemic country like Ghana.

399 Declarations

401 Ethical Approval and Consent to participate

402 Ethics approval for the original clinical trial was obtained from the Ghana Health Service (GHS)

403 Ethical Review Committee, Food and Drugs Authority (FDA) of Ghana, Kintampo Health 404 Research Centre (KHRC) Institutional Ethics Committee Institutional Ethics Committee and 405 Hospital for Sick Children (SickKids) Research Ethics Board, Canada. The secondary analysis of 406 trial data, as well as the primary analysis of immunogenetic data, were approved by the SickKids 407 Research Ethics Board and KHRC Institutional Ethics Committee Institutional Ethics Committee.

408 Informed consent was obtained from each participant's primary caregiver before screening and 409 enrolment in the trial.

\section{Consent for publication}

412 Not applicable.

\section{Availability of data and Materials}

415 The datasets supporting the conclusions of this article are available upon request. 
419 The authors declare that they have no competing interests.

\section{$421 \quad$ Funding}

422 Funding for the original study was provided by the National Institutes of Health (NIH) (grant 423 5U01HD061270-02); Eunice Kennedy Shriver National Institute of Child Health and Human 424 Development (NICHD); Office of Dietary Supplements (ODS); Kintampo Health Research Centre 425 (KHRC). The sponsor of the study had no role in study design, data collection, analysis or 426 interpretation, or writing of the report. But funding for this manuscript as part of my doctoral 427 research programme was not available (Not applicable).

\section{Contributors}

$430 \mathrm{KT}, \mathrm{FA}, \mathrm{BA}, \mathrm{SS}, \mathrm{BA}, \mathrm{TG}$ and KP made primary contributions to overall trial development, design 431 and manuscript writing. KT coordinated the trial under the supervision of FA and KP. KT, LA and 432 DA conducted and managed the laboratory analyses. SA designed the database and data 433 management system. FO and FD conducted the statistical analyses. All the authors reviewed and 434 approved the final paper.

\section{Acknowledgements}

437 We would like to thank the study participants and their caregivers; the KHRC field team and staff; 438 chiefs, opinion leaders and elders of participating communities; participating health facilities; the 439 GHS staff in Wenchi and Tain; the Ethics Boards of KHRC, GHS and SickKids of Canada; the 440 DSMB; and FDA of Ghana. 
$443{ }^{1}$ Department of Biochemistry and Biotechnology, College of Sciences, Kwame Nkrumah 444 University of Science and Technology, Kumasi, Ghana; ${ }^{2}$ Kintampo Health Research Centre, 445 Kintampo-North, Ghana; ${ }^{3}$ Department of Immunology, College of Health Sciences, Noguchi 446 Memorial Institute for Medical Research, University of Ghana, Legon, Accra, Ghana; ${ }^{4}$ Department 447 of Molecular Medicine, School of Medical Sciences, Kwame Nkrumah University of Science and 448 Technology, Kumasi, Ghana; ${ }^{5}$ Department of Biomedical Sciences, School of Allied Health 449 Sciences, University of Cape Coast, Cape Coast, Ghana.

\section{References}

452 1. Black R, Allen L, Bhutta Z, Caulfield L, de Onis M, Ezzati M, Mathers C, Rivera J:

453 Maternal and Child Undernutrition Study Group: Maternal and child 454 undernutrition 1-maternal and child undernutrition: global and regional exposures

2. Walter T: Effect of iron-deficiency anemia on cognitive skills and neuromaturation in infancy and childhood. Food and Nutrition Bulletin 2003, 24:S104-S110.

3. WHO: Guideline: Daily Iron Supplementation in Infants and Children, WHO Guidelines Approved by the Guidelines Review Committee. World Health Organization; 2016.

461 4. WHO: World Health Organization model list of essential medicines: 21 st list 2019. World Health Organization; 2019.

463 5. Horton S, Shekar M, Ajay M: Scaling up nutrition: What will it cost?: The World Bank; 464 2009. 
465 6. Muthayya S, Rah JH, Sugimoto JD, Roos FF, Kraemer K, Black RE: The global hidden 466 hunger indices and maps: an advocacy tool for action. PLoS One 2013, 8.

467 7. Zlotkin SH, Christofides AL, Hyder SZ, Schauer CS, Tondeur MC, Sharieff W: 468 Controlling iron deficiency anemia through the use of home-fortified complementary foods. The Indian Journal of Pediatrics 2004, 71:1015-1019.

470 8. Chang S, Zeng L, Brouwer ID, Kok FJ, Yan H: Effect of iron deficiency anemia in pregnancy on child mental development in rural China. Pediatrics 2013, 131:e755e763.

473 9. Nyakeriga AM, Troye-Blomberg M, Chemtai AK, Marsh K, Williams TN: Malaria and 474 nutritional status in children living on the coast of Kenya. Scandinavian Journal of $475 \quad$ Immunology 2004, 59:615-616.

476 10. Sazawal S, Black RE, Ramsan M, Chwaya HM, Stoltzfus RJ, Dutta A, Dhingra U, Kabole 477 I, Deb S, Othman MK, Kabole FM: Effects of routine prophylactic supplementation with iron and folic acid on admission to hospital and mortality in preschool children in a high malaria transmission setting: community-based, randomised, placebocontrolled trial. Lancet 2006, 367:133-143.

481 11. Murphy SC, Breman JG: Gaps in the childhood malaria burden in Africa: cerebral malaria, neurological sequelae, anemia, respiratory distress, hypoglycemia, and complications of pregnancy. The American journal of tropical medicine and hygiene

485 12. Chandyo RK, Henjum S, Ulak M, Thorne-Lyman AL, Ulvik RJ, Shrestha PS, Locks L, 486 Fawzi W, Strand TA: The prevalence of anemia and iron deficiency is more common 
in breastfed infants than their mothers in Bhaktapur, Nepal. European journal of clinical nutrition 2016, 70:456-462.

489 13. Breman JG: The ears of the hippopotamus: manifestations, determinants, and estimates of the malaria burden. Am J Trop Med Hyg 2001, 64:1-11.

491 14. Miller JL: Iron deficiency anemia: a common and curable disease. Cold Spring Harbor perspectives in medicine 2013, 3:a011866.

493 15. Grantham-McGregor S, Ani C: A review of studies on the effect of iron deficiency on cognitive development in children. The Journal of nutrition 2001, 131:649S-668S.

495 16. Lozoff B, Jimenez E, Hagen J, Mollen E, Wolf AW: Poorer behavioral and developmental outcome more than $\mathbf{1 0}$ years after treatment for iron deficiency in

17. Arija V, Hernández-Martínez C, Tous M, Canals J, Guxens M, Fernández-Barrés S, Ibarluzea J, Babarro I, Soler-Blasco R, Llop S: Association of Iron Status and Intake During Pregnancy with Neuropsychological Outcomes in Children Aged 7 Years: The Prospective Birth Cohort Infancia y Medio Ambiente (INMA) Study. Nutrients 2019, 11:2999.

18. WHO/UNICEF: Iron supplementation of young children in regions where malaria transmission is intense and infectious disease highly prevalent: Joint statement.Geneva, Switzerland: World Health Organization and UNICEF. World Health Organization and UNICEF; 2006.

507 19. De-Regil LM, Suchdev PS, Vist GE, Walleser S, Pena-Rosas JP: Home fortification of foods with multiple micronutrient powders for health and nutrition in children under two years of age. Cochrane database of systematic reviews (Online) 2011, 9:CD008959. 
510 20. GSS: 2010 Population \& Housing Census: Brong-Ahafo Region. Ghana Statistical $511 \quad$ Service; 2013.

512 21. Owusu-Agyei S, Asante KP, Adjuik M, Adjei G, Awini E, Adams M, Newton S, Dosoo

513 D, Dery D, Agyeman-Budu A, et al: Epidemiology of malaria in the forest-savanna $514 \quad$ transitional zone of Ghana. Malar J 2009, 8:220.

515 22. Addy-Tayie N: 3D-Printed Microscope Accessory: Affordable Technology for $516 \quad$ Efficient Diagnostics. 2019.

517 23. WHO: Worldwide prevalence of anaemia 1993-2005. In WHO Global Database on 518 Anaemia. Geneva, Switzerland: World Health Organization; 2008.

519 24. da Rocha Silla LM: Intermittent Preventive Treatment with Dihydroartemisinin520 Piperaquine in Ugandan Schoolchildren Selects for Plasmodium falciparum 521 Transporter Polymorphisms That Modify Drug Sensitivity. Blood Cells Mol Dis 2016, $522 \quad 60: 5649-5654$.

523 25. Dietary Reference Intakes Tables and Application 524 [http://www.iom.edu/Activities/Nutrition/SummaryDRIs/DRI-Tables.aspx]

525 26. Zlotkin S, Newton S, Aimone AM, Azindow I, Amenga-Etego S, Tchum K, Mahama E, 526 Thorpe KE, Owusu-Agyei S: Effect of iron fortification on malaria incidence in infants 527 and young children in Ghana: a randomized trial. JAMA 2013, 310:938-947.

528 27. WHO: Guidelines for the treatment of malaria. World Health Organization; 2015.

529 28. Pepys MB: C-reactive protein fifty years on. Lancet 1981, 1:653-657.

530 29. Verhoef H, West CE, Ndeto P, Burema J, Beguin Y, Kok FJ: Serum transferrin receptor 531 concentration indicates increased erythropoiesis in Kenyan children with 532 asymptomatic malaria. Am J Clin Nutr 2001, 74:767-775. 
533 30. UNICEF: Malaria Prevention and Treatement. In Promoting Rational use of Drugs and $534 \quad$ Correct Case Management in Basic Health Services. New York, USA: UNICEF; 2000.

535 31. Zlotkin S, Antwi KY, Schauer C, Yeung G: Use of microencapsulated iron (II) fumarate 536 sprinkles to prevent recurrence of anaemia in infants and young children at high risk. 537 Bulletin of the World Health Organization 2003, 81:108-115.

538 32. Adam I: Anemia, Iron Supplementation and Susceptibility to Plasmodium falciparum Malaria. EBioMedicine 2016, 14:13-14.

540 33. Ganz T: Hepcidin and iron regulation, 10 years later. Blood 2011, 117:4425-4433.

541 34. Goheen M, Wegmüller R, Bah A, Darboe B, Danso E, Affara M, Gardner D, Patel J,

548 36. Armstrong AL: Anemia in Central-Asia Pre-school Children: Definition, Risk Factors and Evaluation of Home Fortification Intervention. 2009.

550 37. Pasricha S-R, Hayes E, Kalumba K, Biggs B-A: Effect of daily iron supplementation on health in children aged 4-23 months: a systematic review and meta-analysis of randomised controlled trials. The Lancet Global Health 2013, 1:e77-e86.

553 38. Thompson J, Biggs B-A, Pasricha S-R: Effects of daily iron supplementation in 2-to 5554 year-old children: systematic review and meta-analysis. Pediatrics 2013, 131:739-753. 
555 39. Low M, Farrell A, Biggs B-A, Pasricha S-R: Effects of daily iron supplementation in primary-school-aged children: systematic review and meta-analysis of randomized controlled trials. CMAJ 2013, 185:E791-E802.

558 40. Bryszewska MA, Laghi L, Zannoni A, Gianotti A, Barone F, Saa T, Danielle L, Bacci ML, Ventrella D, Forni M: Bioavailability of microencapsulated iron from fortified bread assessed using piglet model. Nutrients 2017, 9:272.

561 41. Prentice AM, Verhoef H, Cerami C: Iron fortification and malaria risk in children. JAMA 2013, 310:914-915.

563 42. Reichert CO, Da Cunha J, Levy D, Maselli LMF, Bydlowski SP, Spada C: Hepcidin: homeostasis and diseases related to iron metabolism. Acta haematologica 2017,

43. Burté F, Brown BJ, Orimadegun AE, Ajetunmobi WA, Afolabi NK, Akinkunmi F, Kowobari $\mathrm{O}$, Omokhodion S, Osinusi K, Akinbami FO: Circulatory hepcidin is associated with the anti-inflammatory response but not with iron or anemic status in childhood malaria. Blood 2013, 121:3016-3022.

44. Mwangi MN, Maskey S, Andang'o PE, Shinali NK, Roth JM, Trijsburg L, Mwangi AM, Zuilhof H, van Lagen B, Savelkoul HF: Diagnostic utility of zinc protoporphyrin to

45. Zimmermann MB, Molinari L, Staubli-Asobayire F, Hess SY, Chaouki N, Adou P, Hurrell RF: Serum transferrin receptor and zinc protoporphyrin as indicators of iron status in African children. The American journal of clinical nutrition 2005, 81:615-623. 
576 46. Teshome EM, Prentice AM, Demir AY, Andang'o PE, Verhoef H: Diagnostic utility of 577 zinc protoporphyrin to detect iron deficiency in Kenyan preschool children: a community-based survey. BMC hematology 2017, 17:11.

579 47. Kanuri G, Chichula D, Sawhney R, Kuriakose K, De'Souza S, Pais F, Arumugam K, Shet AS: Optimizing diagnostic biomarkers of iron deficiency anemia in communitydwelling Indian women and preschool children. haematologica 2018, 103:1991-1996.

48. Zimmermann MB: Methods to assess iron and iodine status. Br J Nutr 2008, 99 Suppl 3:S2-9.

584 49. Ojukwu JU, Okebe JU, Yahav D, Paul M: Oral iron supplementation for preventing or treating anaemia among children in malaria-endemic areas. Cochrane Database Syst Rev 2009:CD006589.

50. Bergdahl B, Bogentoft C, Jonsson UE, Magnusson JO: Fasting and postprandial

51. Olver JS, Burrows GD, Norman TR: The treatment of depression with different formulations of venlafaxine: a comparative analysis. Hum Psychopharmacol 2004,

593 52. Baldi A, Bontempo V, Cheli F, Carli S, Sgoifo Rossi C, Dell'Orto V: Relative bioavailability of vitamin $\mathrm{E}$ in dairy cows following intraruminal administration of

597 53. Menendez C, Fleming A, Alonso P: Malaria-related anaemia. Parasitology today 2000, 16:469-476. 
599 54. Mwangi MN, Roth JM, Smit MR, Trijsburg L, Mwangi AM, Demir AY, Wielders JP, 600 Mens PF, Verweij JJ, Cox SE: Effect of daily antenatal iron supplementation on Plasmodium infection in Kenyan women: a randomized clinical trial. Jama 2015, 314:1009-1020.

603 


\section{Figures}

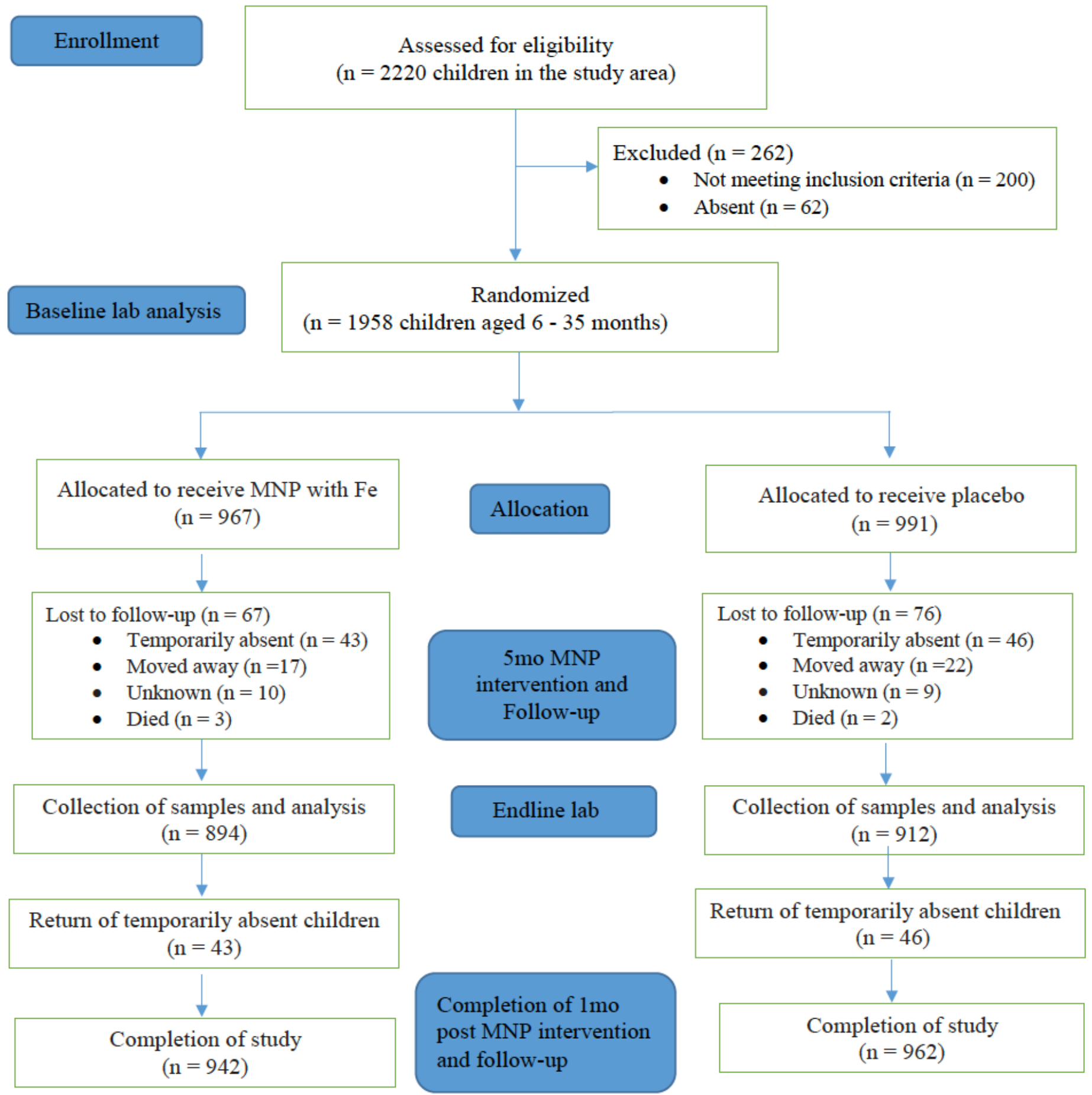

Micronutrient powder (MNP), Fe (iron), Non-Fe (Non-iron), Month (mo), Number of children (n)

${ }^{\mathrm{a}} \mathrm{A}$ cluster represents one or more families with at least a recruited child living from the same compound in the study

\section{Figure 1}

Study profile

\section{Supplementary Files}


This is a list of supplementary files associated with this preprint. Click to download.

- IronstatusTable.pdf 